\title{
Close2U- An Android assistant to show nearby places by using various API service providers
}

\author{
Bille Giriteja ${ }^{1}$, Shivangi Raj ${ }^{2}$, Dr. Usharani ${ }^{3}$ \\ ${ }^{1}$ Student, Dept of Electronics and Communication, R.V. College of Engineering, \\ Bangalore, INDIA \\ ${ }^{2}$ Student, Dept of Computer Science Engineering, P E S University, Bangalore, \\ INDIA \\ ${ }^{3}$ Associate Professor, Dept of Electronics and Communication,R.V. College of \\ Engineering, Bangalore, INDIA \\ 19iriteja94495@gmail.com,2hㅗㄹangiraj902@gmail.com, ${ }^{3}$ usharani@rvce.edu.in
}

\begin{abstract}
With the immense use of android operating systems in today's technology, Android apps became part of our daily lives and we use different apps for different purposes. The motivation behind every location based application is to give the user live experience with real data at the real time. To ease the searching of nearby places for the user, this android application android application provides the details of nearby locations like restaurants, hospitals, emergency services, travel services and also COVID vaccination centres like an assistantthat answers user's query based on different queries. The apps that are available already use location and show the google maps inside the application which makes the application heavy and the cost of API request in that is high, but in this application, the user interface will be simple and it will fetch only the required data from different API Service providers and the data adapters for the application change based on different API service providers.
\end{abstract}

Keywords: Android development, Assistant, Software, APIs,Location,Places,Search,Google,Vaccine

\section{INTRODUCTION}

In Today's generation, there are over 2 Billion active monthly users who uses android devices, more than 1300 brands included android operating system in their devices and there are over 3 million apps in play store.Thus, Android plays a very important role in everyone's day to day life. So it is important for the android developers to create many different applications that fulfils different purpose to ease the life of the user. People shifting to new places may find it difficult to locate nearby restaurants, ATMs,hospitals,gas filling stations etc. Thus this application built on idea of assistant which can be called as Close2U and is an android application created by using Kotlin ,Java,Google Jetpack Compose,Android Studio and various other UI/UX libraries. The purpose of this app is to provide details like 
timings,address,reviews with rating,distance of nearby places. On viewing the present pandemic conditions, this assistant also provides the information like number of vaccine slots, type of vaccines available in the nearby hospitals and cost of the vaccine. All the location based search applications use the User's location and display the data from the API's in the google maps inside the application ,but it is very important to organise the data in a very detailed and confined manner. This project shows the nearby places in the application without using google maps rather uses Google Places API to fetch the data into the UI which is quick to load than google maps in a very customised manner and there are user-friendly choices to filter the data that users can use based on their choice.When Places API doesn't have enough data ,the assistant switches to foursquare API and renders the data from their datasets.

\section{APPLICATION COMPONENTS}

The application comprises of 4 Components

1) Google and Facebook API

2) Geo-Location of user

3) Google Places API

4) Application User Interface

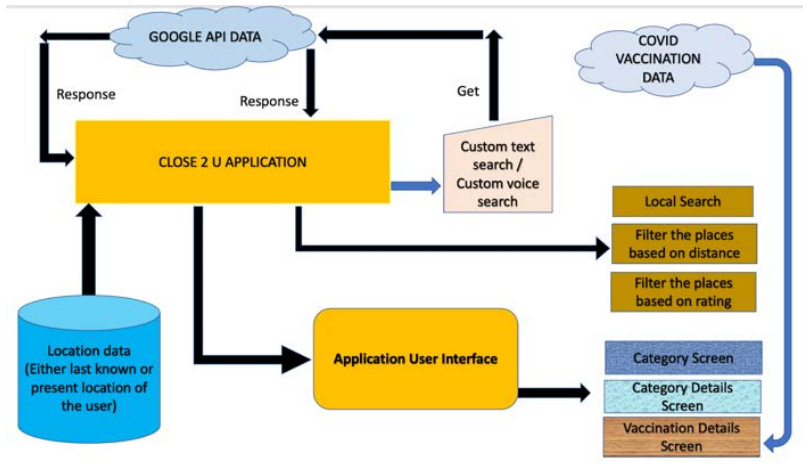

Fig. 1. Application's Architecture

\subsection{Google and Facebook Authentication:}

In this generation, every application has a user authentication feature which helps in detecting the unauthorised user or spam bots who misuses the API requests and overloads the API servers In order to let the valid user to use the application features .This application implements 2 options for Logging in .

1) Sign In With Google

2) Sign In With Facebook

Google and Facebook login uses their respective Access tokens as shown in Fig 1 and hits their API's to verify the user's access token and thus user is prompted to go to the Home Screen and use app's features. If the access token is not created then it will create for the first time and store it in the respective API. 


\subsection{Geo-Location Of User}

In order to make any location based application possible, we have to know the current location or last known location of the android user. There are different Geolocation APIs that provide the current or last known location of the user based on the API requests.This application uses the Fused location provider which is a location API in Google Play services that intelligently combines different signals to provide the location information. It manages the technologies such as GPS and WI-FI by storing their IP addresses. As shown in Fig 1, the last known location of the user is stored inside the phone's internal memory which will be retrieved by the application as and when it is required to perform any API request.

\subsection{Google Places API}

In order to fetch the different places this application uses 3 different Google APIs.

1. Based on the location of the user, this assistant uses Places API, which provides different types of places nearby based on the type of the place and radius in which places should be present.

2. To fetch more details about a certain place, it uses another API which can give sole details of a particular place .Based on the user's custom search or voice search, it fetch the more different places.

3. By making use of a custom query defined by the user. It hits another API with query parameter as the text given by the user.

This application uses google places API,but we can also integrate other different API service providers such as foursquare or any other API which would give a similar format of the required data with our android application.

The data provider may differ but the assistant adapts to different API service providers.

\subsection{Application's User Interface}

Structuring the data we fetch from different APIs in a well defined manner where the user can have the best experience with the application has always been an important task for the android and UI developers. In this assistant it uses a simple and attractive design for the User Interface which would take very less time to load and also creates a good impact for the user.The API rendering is done without using any available libraries and implemented the UI and API by using Async Task in android. The UI is built by using XML and different UI/UX android libraries. 


\section{PROPOSED MODEL}

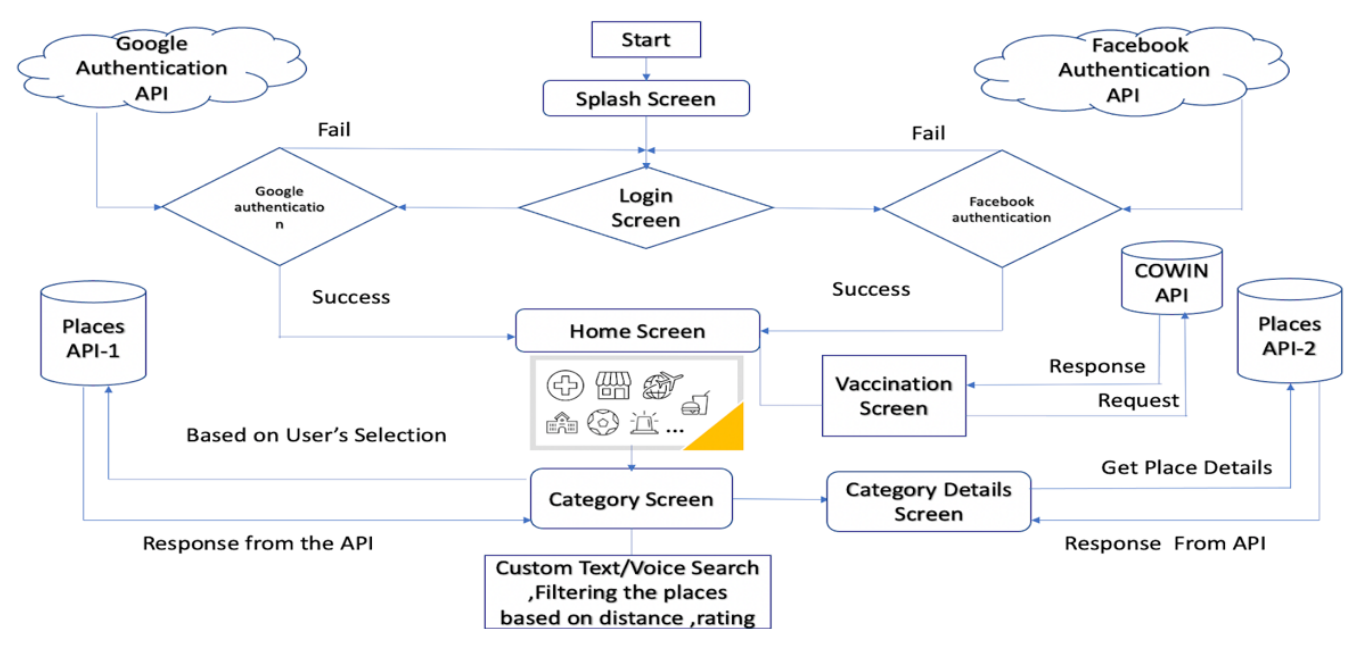

Fig. 2. The Flow Chart

The model is built based on the fig 2 as shown above. This Application consist of the following different screens:

- The Splash Screen

- The Login Screen

- The Home Screen

- The Category Screen

- The Category Details Screen

- Navigation Menu Screen

- The Vaccine Details Screen

\section{The Splash Screen :}

The splash screen is the start-up screen which appears when the app is opened. It gives a small overview about thisassistant. The time frame in which splash screen is shown in the application is enough for all the background tasks,such as checking whether user has actually logged in using Facebook or Google .If the user is not logged in with any of the methods, then it opens the Login Screen. The Splash Screen is shown in fig below. 


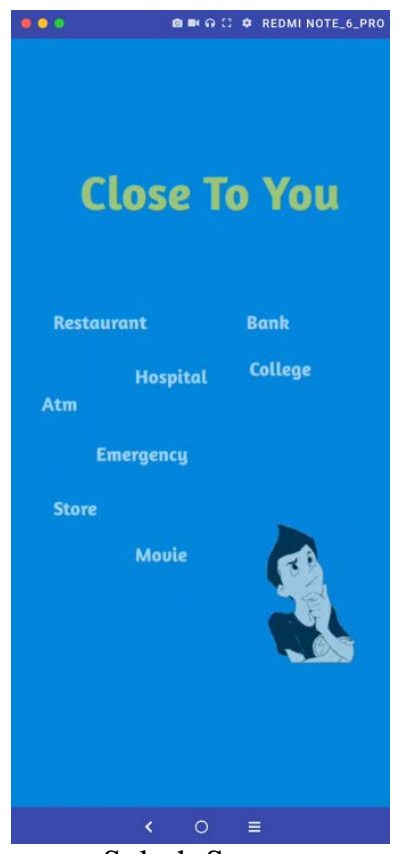

Splash Screen

\section{The Login Screen :}

This Screen consists of two types of Authentication, Google and Facebook. Users may login through any one of the authentication.

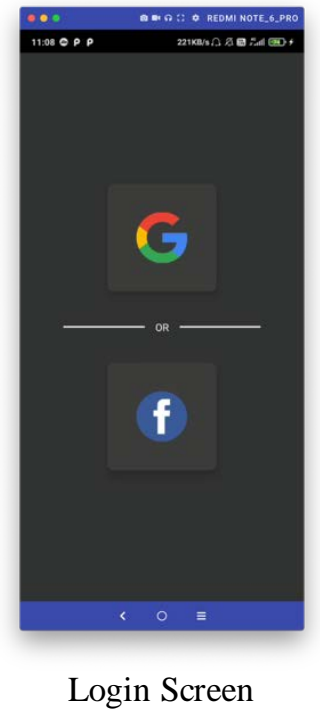

\section{The Home Screen :}

This is the main screen of this assistant which consists of 9 different options. Each Option represents a category like restaurants, fun, hospital, stores, service, sports, travel, education etc. On clicking any button like hospital, the user will be shown a list of nearby hospitals, their distance from the current user's location and images if available. All the hospitals up to $5 \mathrm{kms}$ are shown .Similarly it does the same function for other 8 options. The Home Screen also consists of the custom text search and also voice search button which lets users search based on their text or voice input. It also contains an icon whose purpose is to show all the 
nearby hospitals where COVID vaccine slots are available and details regarding the hospital and. also the vaccine availability

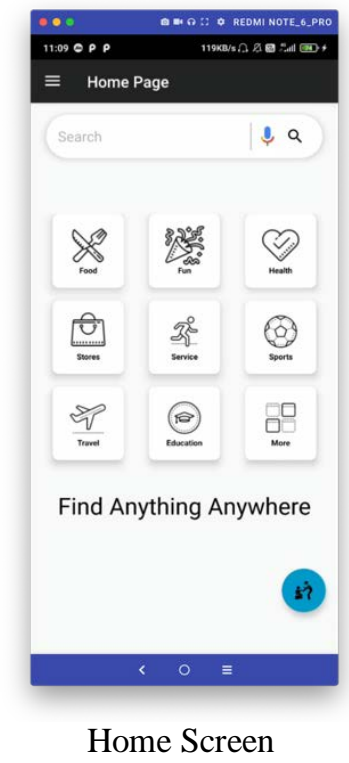

\section{The Category Screen :}

This Screen consists of the list of nearby places up to 5 kilometres. Each individual place includes name, images, distance from the current location, favourite icon and the rating of the place. All the places are clickable and on clicking, it opens a new Screen which provides all the details of that particular place. The category screen has the option to sort the places by clicking on the sort by option which is provided on the top of the screen.

On clicking the option ,the user will get 2 options : distance, rating. They reformat the list of places based on distance and rating. Thisassistant also includes a local search through which user can search a particular place in that list of nearby places and refactor the places inside the category screen.

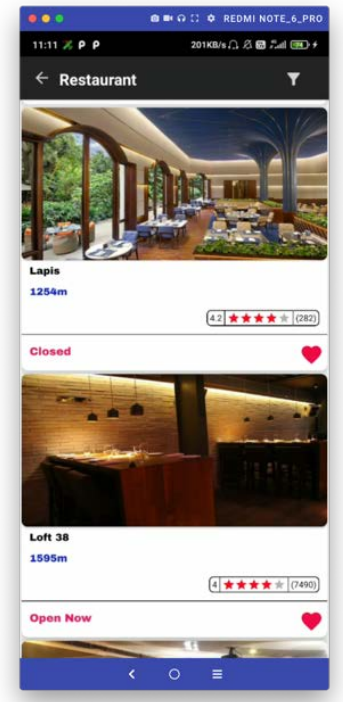

Category Screen 


\section{The Category Detail Screen :}

This is the screen which contains all the details of one particular place. On clicking any one item in the category screen, the user will be shown from the user's current location,Address,rating of the place, icon to add it to the user's favorite, Opening and closing hours on a weekly basis. At last it consists of the reviews given by different users/customers who have visited the place along with their individual rating. This Screen also contains the information of the expected time taken by the user to reach that particular place if the mode of travel is either by walk,bike,or car. This Screen also has the options to navigate to the place from the user's location. It displays the telephone number and also their website(if available). The User can also share that place information via different social media applications.

To navigate directly to the home screen the user is provided with quick button.

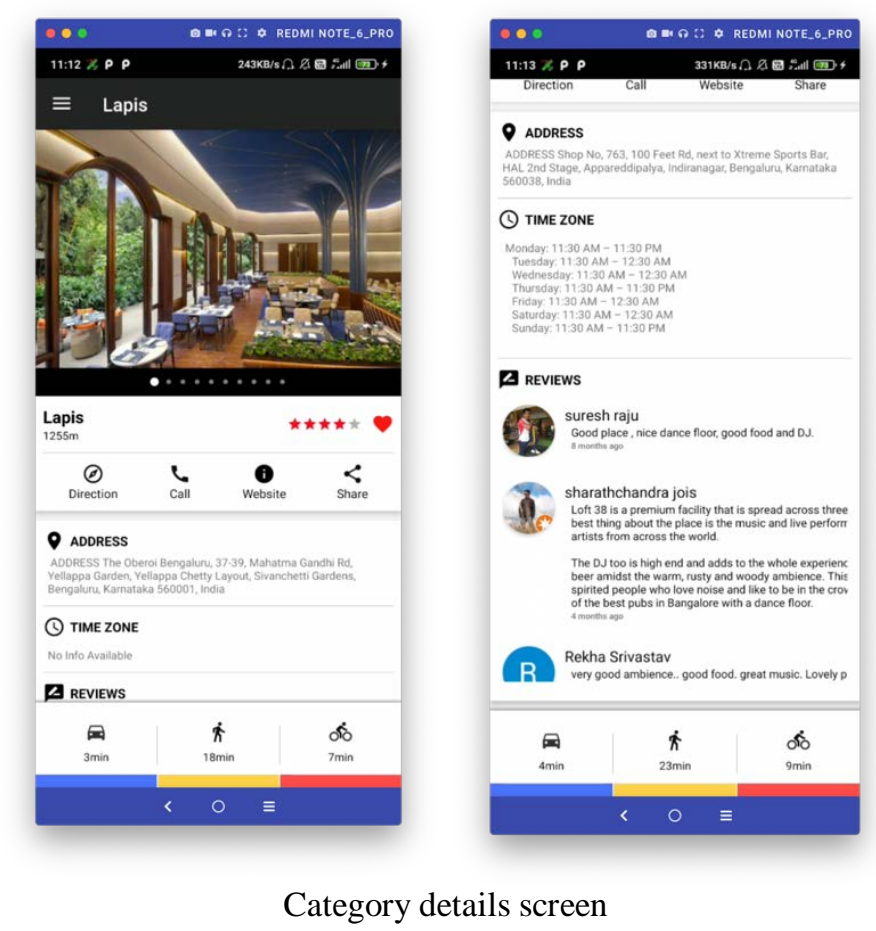

\section{The Navigation Screen :}

There is a hamburger icon on the top-left corner of the Home Screen and Category Details Screen. On clicking this icon the user may visit our navigation view. The view has the user's profile picture, user's name and his registered email ID. This may give a proof that the valid user is logged in successfully. It consists of : Home Page, App Info, Contact Us, Logout.

1) Home Page is used to bring the user back to the Home Screen.

2) App Info gives the details of the functioning of the App.

3) Contact Us contains the developers contact details where the user may submit any specific queries 
4) By clicking the Logout the user will be logged-out from the application.

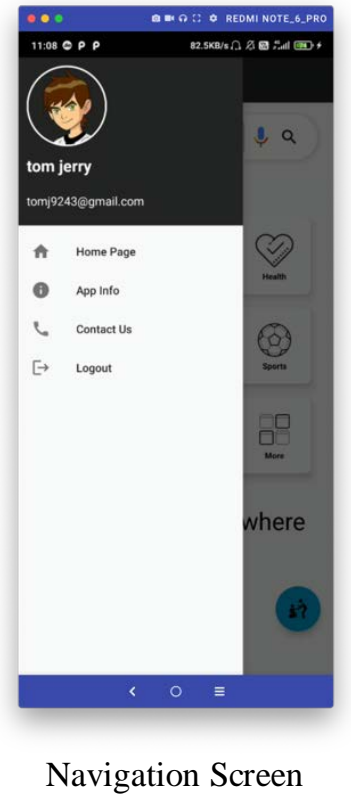

\section{The Vaccine Detail Screen :}

During this pandemic situation, everyone needs to know the correct information about the vaccination centres and also the availability of the vaccine, this screen will take user's input i.e., State and District and gives all the available vaccination centres and also displays the availability of the vaccine and gives detailed information of the vaccination centre.

This screen is shown inside the health category screen with a floating action button, thus on clicking the floating action button the user will be redirected to the vaccination screen where user is asked to choose the State an District .Finally user is provided with the following screens
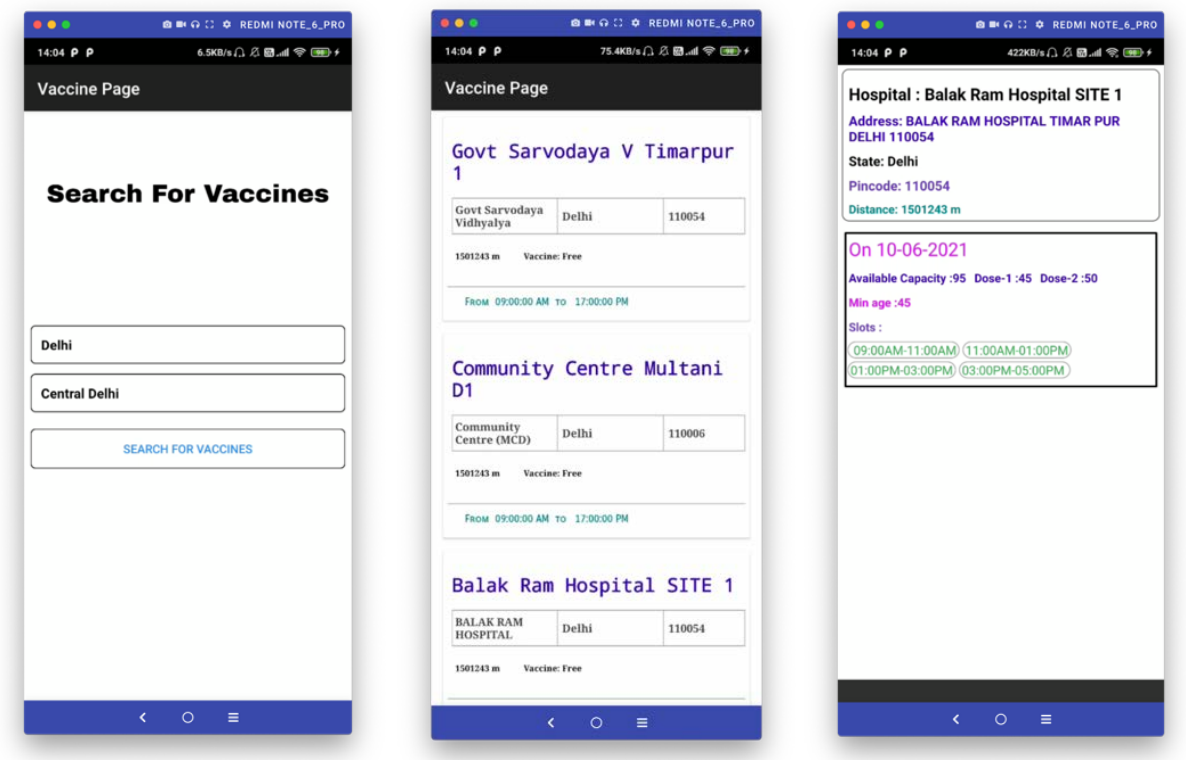
Vaccination screens

\section{4 .CONCLUSION}

Mobile Applications can be developed on Android platform as well as IOS platform. We chose the android as there are many android users compared to IOS users and moreover android is parallel to IOS. Android is provided by google ,by using their simulated environment and also their development kits, we developed an android application using kotlin,android studio and also different API Service providers like Google Place APIs,Cowin APIs which can help user to find nearby places like hospitals,school,stores,gas stations,restaurants,vaccination centers, any other facility of interest indicated by user within certain range. The retrieved results get changed as the user changes his/her position.It also provides the details about COVID vaccination centre and also vaccine slots available in nearby hospitals. Our main motive behind building this application was that the user may know what places are around them. There are fewer apps which only provide information about nearby restaurants, but in this application the user can know more details about the single place as well as he can search for any type of place nearby

\section{FUTURE WORK}

This application shows full details about all the nearby places. For now this app is made only for the android user but in future can also be used for IOS devices. We may add features like booking an appointment in a hospital or booking a table in a restaurant and alert the user about the appointments before they are closed. By using machine learning techniques we can extend the features of the application by rendering the places based on the past visit history of the user, and also the application can act like an assistant which would actively interact with the user like existing voice assistants like Alexa and Siri, where it processes the audio of the user and extracts the keywords by using NLTK and also by using neural networks to put the query parameters inside the API call thus this can respond to the user with the answer. We can also add features such as detecting the mood of the user by doing emotional analysis. The idea behind emotional analysis of the user's face would be to consider whether the user is happy or sad ,based on the mood ,the algorithm can filter the places based on the customer's reviews and render them to the application user.

\section{REFERENCES}

[1]. T. Sohan, K. A. Li, G. Lee, I. Smith, J. Scott, and W. G.Griswold,"Place-Its: A Study of Location-Based Reminders on Mobile Phones,"Proc. 7th Int'l Conf. Ubiquitous Computing (UbiComp2005), LNCS 3660,2005.

[2]. Sandeep Kumar, Mohammed Abdul Qadeer and Archana Gupta, "Location Based Services using Android", IEEE, 2009.

[3].Location Manger API-Google developer documentation,

'http://developer.android.com/reference/android/location.'

[4]. Places API, 'https://developers.google.com/maps/documentation/places/“

[5]. R. Nicole, “Title of paper with only first word capitalized,” J. Name Stand. Abbrev., in press. 
[6]. Y. Yorozu, M. Hirano, K. Oka, and Y. Tagawa, "Electron spectroscopy studies on magneto-optical media and plastic substrate interface,” IEEE Transl. J. Magn. Japan, vol. 2, pp. 740-741, August 1987 [Digests 9th Annual Conf. Magnetics Japan, p. 301, 1982].

[7]. Consortium, O. G. Open location services 1.1, 2005.

[8]. Minh Huynh and Prashant Ghimire "BROWSER APP APPROACH: CAN IT BE AN ANSWER TO THE CHALLENGES IN CROSS-PLATFORM APP DEVELOPMENT?", Journal of information technology education , 2017.

[9]. Neha Verma, Sarita Kansal and Huned Malvi, "Development of Native Mobile Application Using Android Studio for Cabs and Some Glimpse of Cross Platform Apps", International Journal of Applied Engineering Research ,2018.

[10]. Mrs. Prachi Sasankar1 and Mrs. Usha Kosarkar2, "Research on Development of Android Applications", IOSR Journal of Computer Engineering (IOSR-JCE), 2016.

[11]. J. Liu and J. Yu, "Research on development of android applications," in $20114^{\text {th }}$ International Conference on Intelligent Networks and Intelligent Systems, 2011,pp. 69-72. doi: 10.1109/ICINIS.2011.40.

[12]. Y. Yan, S. Cosgrove, V. Anand, A. Kulkarni, H. Sree, S. Konduri, L. Ko, and L. Ziarek, "Real-time android with rtdroid,” Jun. 2014. doi: 10.1145/2594381.

[13].D. E. Gajanan, G. G. Shankar, and G. V. Keshav, "Detection of leaf disease using feature extraction for android based system," International Journal of ScientificResearch in Science and Technology, vol. 4, pp. 450-456, 2018.

[14]. H. Al Rekhawi and S. Abu-Naser, "Android applications ui development intelligenttutoring system," Jan. 2018.

[15]. L. Fetni, Development of a mobile application for carpooling the elderly, Jun. 2019.doi: 10.13140/RG.2.2.22674.20166.

[16].L. Ma, L. Gu, and J. Wang, "Research and development of mobile application forandroid platform," International Journal of Multimedia and Ubiquitous Engineering, vol. 9, pp. 187-198, Apr. 2014. doi: 10.14257/ijmue.2014.9.4.20.

[17]. S. Jagtap and D. Hanchate, "Survey on development of android based mobile app for prestashop ecommerce shopping cart (alc),” International Journal of Modern Computer Science (IJMCS), vol. 5, pp. 2320-7868, Jun. 2017.

[18].M. Latif, Y. Lakhrissi, E. H. Nfaoui, and N. Es-Sbai, "Cross platform approach formobile application development: A survey,” Mar. 2016, pp. 1-5. doi: 10.1109/IT4OD.2016.7479278. 